\title{
Manual de apoyo docente para la evaluación del aprendizaje mediante la utilización de medios de enseñanza
}

\section{Teaching support manual for the evaluation of learning through the use of teaching aids}

\author{
Dra. C Anabel González Sánchez. ${ }^{1}$, Lic. Fernando Pujol Bosque. ${ }^{2}$, Lic. Yaneisi Sánchez \\ Sierra. $^{3}$ \& MSc. Ana María Sánchez Silva ${ }^{4}$
}

\begin{abstract}
.
The evaluation of learning is an essential part of the teaching-learning process that enables us to direct it, as well as the control and evaluation of the modes of action that students acquire through the development of the teaching process, when verifying the degree with the one that the proposed objectives are reached. The objective of this research is to develop a teaching support manual for the evaluation of learning
\end{abstract}

\footnotetext{
${ }^{1}$ Universidad de Ciencias Médicas de Matanzas. Facultad de Ciencias Médicas "Dr. Juan Guiteras Gener", Matanzas, Cuba, anabelg.mtz@infomed.sld.cu, (으 https://orcid.org/0000-0002-3528-5961

${ }^{2}$ Universidad de Ciencias Médicas de Matanzas. Filial de Ciencias Médicas "Dr. Eusebio Hernández Pérez", de Colón, Departamento Metodológico. Matanzas, Cuba, fer@fcmc.sld.cu, (1) https://orcid.org/0000-00033269-007x

${ }^{3}$ Universidad de Ciencias Médicas de Matanzas. Facultad de Ciencias Médicas "Dr. Juan Guiteras Gener. Departamento de Enfermería. Matanzas, Cuba, yaneisysierra.mtz@infomed.sld.cu, () https://orcid.org/00000003-3649-5175

${ }^{4}$ Universidad de Ciencias Médicas de Matanzas. Filial de Ciencias Médicas "Dr. Eusebio Hernández Pérez". Matanzas, Cuba, anamariasanchezsilva@fcmc.sld.cu, (1) https://orcid.org/0000-00031866-9434
} 
through the use of teaching aids. A development research was carried out at the Matanzas University of Medical Sciences during the school years 2017 - 2018 to 2018 - 2019. Methods of the theoretical level were used: analytical-synthetic and inductivedeductive and systemic-structural; and empirical: documentary analysis of the analytical programs of the subjects that make up the Nursing discipline and methodological guidelines, observation of classes, the survey of teachers and specialists. It was evident that there are no specific orientations in the analytical programs of the subjects that make up the Nursing discipline for the development of the evaluation of learning through the use of teaching means; insufficiencies in the learning evaluation process were detected by teachers using traditional and schematic methods, lack of correspondence of questions, objectives to be evaluated and levels of assimilation, and the potentialities of the teaching means for evaluating the learning. A teaching support manual was prepared to help solve the deficiencies detected. The manual was valued by specialist criteria as pertinent, novel, useful and was a valuable tool for the self-preparation of teachers and stimulating developer learning in students.

Keywords: manual, teaching aids, evaluation, developer learning.

\section{Resumen.}

La evaluación del aprendizaje es una parte esencial del proceso de enseñanzaaprendizaje que nos posibilita su propia dirección, así como el control y la valoración de los modos de actuación que los estudiantes adquieren a través del desarrollo del proceso docente, al comprobar el grado con el que se alcanzan los objetivos propuestos. El objetivo de la presente investigación es elaborar un manual de apoyo docente para la evaluación del aprendizaje mediante la utilización de medios de enseñanza. Se realizó una investigación de desarrollo en la Universidad de Ciencias Médicas de Matanzas durante los cursos escolares 2017 - 2018 a 2018 - 2019. Se utilizaron métodos del nivel teórico: analítico-sintético e inductivo-deductivo y sistémico - estructural; y empíricos: análisis documental de los programas analíticos de las asignaturas que conforman la disciplina Enfermería y las orientaciones metodológicas, observación de clases, la encuesta a profesores y especialistas. Se evidenció que no existen orientaciones específicas en los programas analíticos de las asignaturas que integran la disciplina Enfermería para el desarrollo de la evaluación del aprendizaje mediante la utilización de medios de enseñanza; fueron detectadas insuficiencias en el proceso de evaluación del aprendizaje por los docentes empleo de métodos tradicionales y esquemáticos, falta de correspondencia de las preguntas, objetivos a evaluar y los niveles de asimilación y no se aprovechan las potencialidades de los medios de enseñanza para la evaluación del aprendizaje. Se elaboró un manual de apoyo docente que contribuye a solucionar las insuficiencias detectadas. El manual 
fue valorado por criterio de especialistas como pertinente, novedoso, útil y constituyó una herramienta valiosa para la autopreparación de los profesores y estimular el aprendizaje desarrollador en los estudiantes.

Palabras claves: manual, medios de enseñanza, evaluación, aprendizaje desarrollador.

\section{Introducción.}

Desde épocas inmemoriales el hombre ha sentido la necesidad de conocer y trasladar los conocimientos adquiridos, auxiliados de diferentes medios para lograr y facilitar la comprensión de lo que desea trasmitir o enseñar. Se definen como recursos materiales que inciden en la transmisión educativa, favorecen la comunicación entre profesores y alumnos, y solo tienen sentido cuando se conciben en relación con el aprendizaje. (Espín JC, Abad JC, Báez EG, Fernández J \& Cardona A, 2010; Gutiérrez, M., López, R., Yanes, R., Llerena, M., Rosa, M., \& Olano, M, 2013; Mugarra, CG, Pérez, H \& Bujardón, A, 2011).

Las exigencias del desarrollo hacen necesaria la búsqueda y experimentación de nuevas formas de impartir la docencia teniendo como eje del proceso de enseñanza aprendizaje al sujeto que aprende, al procurar que sea significativo para él y le propicie una educación que le dé un especial valor a los aspectos éticos y morales, al asumir como base cuatro pilares fundamentales: aprender a aprender, aprender a actuar, aprender a vivir juntos y aprender a ser. De ahí se deduce la importancia de formar un hombre nuevo; protagonista y responsable de su aprendizaje como participante activo, reflexivo y valorativo capaz de elaborar estrategias en función de los objetivos que se trazan con alto grado de cientificidad.

El aprendizaje es toda actividad del estudiante cuyo resultado es la formación y la adquisición de nuevos conocimientos, habilidades, hábitos y modos de actuación, así como el perfeccionamiento de los que ya se poseían. (Martínez CV, Barrera JL, Baralt D \& Serrano J, 2011)

En los modos de actuación se estructuran e integran los elementos de la personalidad desarrollados en el estudiante, así como el sistema de conocimientos, habilidades y hábitos de su formación profesional (Salas, RS, 1998) que se va a manifestar en la práctica social por una actitud consciente y responsable en su aplicación de manera creadora y de forma independiente. 
La evaluación del aprendizaje es una parte esencial del proceso actual de enseñanza aprendizaje, que posibilita su propia dirección, así como la atención a las individualidades de cada educando, al valorar lo alcanzado en los modos de actuación profesional, prevista para cada etapa del programa de estudio. (Salas, RS \& Salas, A, 2018)

Toda evaluación siempre estará dirigida a verificar el aprendizaje alcanzado en correspondencia con el sistema de objetivos educacionales propuestos. Pero la experiencia indica que el desarrollo del proceso de enseñanza por los profesores influye de forma manifiesta en lo que los educandos estudian y aprenden. Así, la evaluación "arrastra" el aprendizaje a través de los siguientes aspectos: método de enseñanza utilizado; contenido objeto de evaluación; formato de las preguntas evaluativas empleadas y materiales didácticos empleados. (Salas, RS \& Salas, A, 2017)

En clases observadas a profesores que imparten diferentes asignaturas que integran la disciplina Enfermería de la carrera Licenciatura en Enfermería se detecta que utilizan métodos de evaluación tradicionales que no estimulan la evaluación del aprendizaje de sus estudiantes, por lo que se hace necesario la búsqueda de otras vías o alternativas que faciliten la evaluación de los contenidos impartidos de forma asequible e interactiva, lo que permitirá que se enfrenten a situaciones simuladas que le den salida a las situaciones reales de aprendizaje en los escenarios docentes donde se desempeñarán como futuros profesionales de la enfermería.

Hecho que motivó a los autores de la presente investigación a elaborar un manual de apoyo docente para la evaluación del aprendizaje mediante la utilización de medios de enseñanza.

\section{Metodología.}

Se realizó una investigación de desarrollo en la Universidad de Ciencias Médicas de Matanzas durante los cursos escolares 2017 - 2018 a 2018 - 2019. El universo estuvo constituido por 18 profesores del departamento de Enfermería que integran el colectivo de la disciplina Enfermería de la carrera Licenciatura en Enfermería.

Se utilizaron métodos de los niveles teórico y empírico. Del nivel teórico: analítico-sintético e inductivo-deductivo los cuales facilitaron la argumentación de la investigación y la interpretación de los resultados y el sistémico-estructural para garantizar la coherencia y estructuración interna del documento elaborado. 
Del nivel empírico y según el principio ético de voluntariedad fueron aplicados: Análisis documental de los programas analíticos que integran la disciplina Enfermería y las orientaciones metodológicas correspondientes.

La observación de clases a los 18 profesores que componen el colectivo de la disciplina Enfermería con el objetivo de identificar las insuficiencias en la evaluación del aprendizaje. La encuesta a los 18 profesores de la disciplina permitió conocer sus criterios acerca de las principales dificultades en la evaluación del aprendizaje.

Se solicitaron criterios de 15 profesores para valorar la propuesta del manual de apoyo por criterio de especialistas, mediante un cuestionario semiestructurado. Entre ellos ocho doctores en ciencias: seis en Ciencias Pedagógicas, uno en Ciencias de la Salud, uno en Ciencias de la Enfermería y siete másteres en Ciencias de la Educación Superior, con categoría docente de titular y auxiliares respectivamente y con experiencia en la docencia médica de más de 20 años. Los indicadores de la valoración estuvieron relacionados con el nivel de actualización de la propuesta, su novedad, utilidad y pertinencia.

La valoración del manual de apoyo docente incluyó los siguientes aspectos:

- Pertinencia del manual.

- Correspondencia entre el objetivo y los contenidos del manual de apoyo docente.

- Necesidad de la propuesta.

- Importancia en el contexto de la carrera de Licenciatura en Enfermería.

- Utilidad y factibilidad del manual de apoyo docente para ser utilizado en la evaluación del aprendizaje mediante la utilización de medios de enseñanza.

- Rigor científico.

Para ello se estableció la siguiente escala de valoración constituida por cinco categorías: C1 (Muy adecuado), C2 (Adecuado), C3 (Poco adecuado) y C4 (Inadecuado).

Se consideró aceptado si más del $90 \%$ de las consideraciones de los especialistas se encontraban dentro de las categorías de muy adecuado y adecuado. Para el análisis de los resultados se utilizó la estadística descriptiva para el cálculo de las frecuencias absolutas y el porcentaje. 


\section{Resultados.}

A partir del análisis crítico de los programas analíticos que integran la disciplina y las orientaciones metodológicas se evidenció que no existen orientaciones específicas para el desarrollo de la evaluación del aprendizaje mediante la utilización de medios de enseñanza. En clases observadas a los profesores de la carrera de Licenciatura en Enfermería que imparten docencia en las diferentes asignaturas que integran la disciplina Enfermería se identificaron las siguientes dificultades: los docentes utilizan métodos de evaluación tradicionales que no estimulan la evaluación del aprendizaje de sus estudiantes; en ocasiones las preguntas no se corresponden con los objetivos a evaluar y con los niveles de asimilación; y no se aprovechan las potencialidades que ofrecen los medios de enseñanza para la evaluación del aprendizaje. Lo expresado con anterioridad está en correspondencia con estudios de Salas RS (1998) cuando plantea que “... en la práctica todo esto se traduce en preguntas improvisadas que no están acordes con los objetivos a evaluar; ejercicios y problemas que no se corresponden con los niveles de asimilación propuestos..."

Los autores coinciden con Rodríguez JD, Pernas M, Lima L, Díaz M, Montano JA, Pérez G (2011) cuando plantean que los medios de enseñanza adquieren una connotación cualitativamente diferente dentro del proceso docente - educativo, convirtiéndose realmente en una categoría didáctica, como componentes que pueden hacer más rápido y efectivo el aprendizaje, que pueden disminuir el agotamiento intelectual de los estudiantes, sintetizar un gran volumen de información y hacer mucho más grato y productivo el trabajo de los profesores.

Los medios constituyen uno de los componentes del proceso docente educativo que favorece la asimilación de los conocimientos de los estudiantes. Además, actúan como vía de comunicación y sirven de soporte a los métodos de enseñanza para posibilitar el logro de los objetivos planteados; reducen considerablemente el tiempo dedicado al aprendizaje, porque objetivan la enseñanza; se aprovechan en mayor grado las potencialidades de los órganos sensoriales; se logra una mayor permanencia en la memoria de los conocimientos; elevan la efectividad y motivación del aprendizaje; posibilitan hacer más objetivos los contenidos de cada materia de estudio, y por tanto, lograr mayor eficiencia en el proceso de asimilación del conocimiento por los estudiantes. (Escudero, JM, 1983; Guzmán Y, Ferrer, M, 2012; Bello, 
N, Fentón, M, 2009; Colectivo de autores, 2003; Salas, RS, 1998; Puig, R, González, A, 2012 \& Marrero y otros, 2016).

La encuesta a profesores de la disciplina corroboró que los métodos de evaluación que emplean son tradicionales y esquemáticos.

El diagnóstico anterior avala la necesidad de elaborar un manual de apoyo docente que constituya una herramienta para la evaluación del aprendizaje mediante la utilización de medios de enseñanza.

El manual de apoyo docente está estructurado por una introducción donde explica su objetivo, importancia y utilidad para la evaluación del aprendizaje mediante la utilización de medios de enseñanza en la Educación Médica y posee, además, cuatro capítulos los cuales están organizados de la siguiente forma:

- Capítulo I: Generalidades de los medios de enseñanza y de la evaluación del aprendizaje. En este capítulo además de abordar los conceptos esenciales de medios de enseñanza y evaluación del aprendizaje, se da a conocer la importancia y características de ambos componentes del proceso enseñanza aprendizaje para la Educación Médica.

- Capítulo II: La evaluación como componente regulador del proceso enseñanza aprendizaje. Para su desarrollo se enfatizará en la importancia que juega la evaluación como componente en la comprobación de los conocimientos adquiridos por los estudiantes durante el proceso enseñanza aprendizaje.

- Capítulo III: Indicaciones metodológicas para utilizar los medios de enseñanza en función de la evaluación del aprendizaje.

Aparecen las indicaciones metodológicas dirigidas a los profesores para la utilización de los medios de enseñanza en función de la evaluación del aprendizaje teniendo en cuenta las etapas de planificación, orientación, ejecución y control de la actividad; además se explican las ventajas y desventajas de su empleo para promover un aprendizaje desarrollador.

- Capítulo IV: Medios de enseñanza utilizados para la evaluación del aprendizaje. En el presente capítulo se describirá la metodología de cuatro medios de enseñanza utilizados para evaluar el aprendizaje en diferentes asignaturas que integran la disciplina de Enfermería de la carrera de Licenciatura en Enfermería cuyos resultados potenciaron la adquisición y asimilación de conocimientos de los estudiantes. Los medios de enseñanza empleados se nombran: flor de la integralidad, la suerte es loca, tablero didáctico y domina y aprende. 
El manual de apoyo fue valorado por el $100 \%$ de los especialistas entre muy adecuado y adecuado, considerándolo una herramienta valiosa para los profesores. Opinaron que está actualizado en sus contenidos, es útil y pertinente porque brinda opciones a la solución precisada en esta problemática. Las recomendaciones expresadas facilitaron una mejor conformación del manual.

El manual de apoyo docente aporta beneficios para los docentes que repercuten de forma positiva en el proceso enseñanza aprendizaje al propiciar que este sea interactivo, donde se construya el conocimiento y el estudiante pueda desarrollar sus propias estrategias de aprendizaje.

\section{Conclusiones:}

- El manual de apoyo docente elaborado fue valorado por criterio de especialistas como pertinente, novedoso, útil y constituyó una herramienta valiosa para la autopreparación de los profesores proporcionándole variados ejemplos para su implementación mediante la utilización de medios de enseñanza en las evaluaciones sistemáticas y parciales, así como un instrumento que estimula en los estudiantes un aprendizaje desarrollador.

\section{Referencias bibliográficas.}

Espín Falcón, JC., Abad Araújo, JC., Báez Pérez, EG., Fernández Morín, J., \& Cardona Almeida, A. (2010). Los medios de enseñanza en la orientación de los contenidos en la asignatura Morfofisiología Humana I en el Nuevo Programa de Formación de Médicos en Cuba. Educación Médica Superior, 24(4) Recuperado de: http://scielo.sld.cu/scielo.php?script=sci_arttext\&pid=S086421412010000400001\&lng=es\&tlng=es.

Gutiérrez Escobar, M., López Fernández, R., Yanes Seijo, R., Llerena Bermúdez, M., Rosa Rodríguez, M., \& Olano Rivalta, M. (2013). Medios de enseñanza con nuevas tecnologías versus preparación de los docentes para utilizarlos. Medisur, 11(2), 167 175. Recuperado de: http://medisur.sld.cu/index.php/medisur/article/view/2238/1167

Mugarra Romero, CG., Pérez Rodríguez, H., \& Bujardón Mendoza, A. (2011). Consideraciones sobre la educación en valores a través de los medios de enseñanzaaprendizaje. Humanidades Médicas, 11(3), 538-558. Recuperado de: http://scielo.sld.cu/scielo.php?script=sci_arttext\&pid=S1727-

$81202011000300009 \& \operatorname{lng}=\mathrm{es} \&$ tlng=es

Martínez Bonne, CV., Barrera Romero, JL., Baralt Ramos, D., \& Serrano Savigne, J. (2011).

La preparación de los profesores en medios de enseñanza durante su formación inicial 
un reto de los colectivos pedagógicos de años. Cuadernos de Educación y Desarrollo, 3(25). Recuperado de: http://www.eumed.net/rev/ced/25/brrs.htm.

Salas Perea RS. (1998) La evaluación en la educación superior contemporánea. La Paz, Bolivia: Biblioteca de Medicina. Volumen XXIV. UMSA.

Salas Perea, RS., \& Salas Mainegra, A. (2018) Evaluación para el aprendizaje en ciencias de la salud. La Habana: Editorial Ciencias Médicas. Cuba.

Salas Perea, RS., \& Salas Mainegra, A. (2017) Evaluación para el aprendizaje en ciencias de la salud. Edumecentro, 9(1). Recuperado de: http://www.revedumecentro.sld.cu

Rodríguez Milera, JD., Pernas Gómez, M., Lima Sarmiento, L., Díaz Molleda, M., Montano Luna, JA., \& Pérez Esquivel, GJ. (2011). Valoración de la utilización de los medios de enseñanza-aprendizaje en las actividades de orientación de contenido. Educación Médica Superior, 25(4), 496-514. Recuperado de: http://scielo.sld.cu/scielo.php?script=sci_arttext\&pid=S086421412011000400010\&lng=es\&tlng=es

Escudero Muñoz JM. (1983). La investigación sobre medios de enseñanza: revisión y perspectivas actuales. Recuperado de: http://gredos.usal.es/jspui/bitstream/10366/69155/1/La_investigacion_sobre_medios _de_enseñanza.

Guzmán León, Y., Ferrer Llanes, M. (2012) Los medios de enseñanza y aprendizaje y su contribución a la apropiación de los contenidos de las ciencias naturales. Revista IPLAC, 16(1). Recuperado de: http://www.revista.iplac.rimed.cu/index.php?optio.=com

Bello N, Fentón M. (2009) Proceso enseñanza aprendizaje: Temas para enfermería. La Habana: Editorial Ciencias Médicas.

Colectivo de autores. (2003) Preparación pedagógica integral para profesores universitarios. Instituto Superior Politécnico José Antonio Echeverría, Cuba.

Salas Perea RS. (1998) Los medios de enseñanza en la educación en salud. Biblioteca de Medicina Volumen XXIII. La Paz: Universidad Mayor de San Andrés.

Puig Washington R., \& González Hourruitiner, A. (2012) Criterios de clasificación y selección de los medios de enseñanza. Educación Médica Superior, 26(2), 343-349. Recuperado de: http://scielo.sld.cu/scielo.php?script=sci_arttext\&pid=S0864$21412012000200015 \& \operatorname{lng}=$ es\&tlng=es

Marrero y otros. (2016) Las imágenes digitales como medios de enseñanza en la docencia de las ciencias médicas. Recuperado de: http://www.scielo.org.co/scielo.php?pid=S012312942010000200007\&script=sci_art text

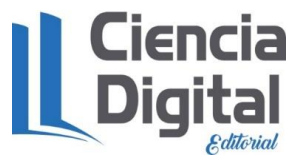




\section{PARA CITAR EL ARTÍCULO INDEXADO.}

Saumell Lafargue , C., \& Hernández Rodríguez, G. C. (2020). Propuesta de Desarrollo para el Turismo de Aventura en el territorio de la Llanura Habana-Matanzas en los límites de las provincias La Habana y Mayabeque. Explorador Digital, 4(3), 140-158. https://doi.org/10.33262/exploradordigital.v4i3.1314

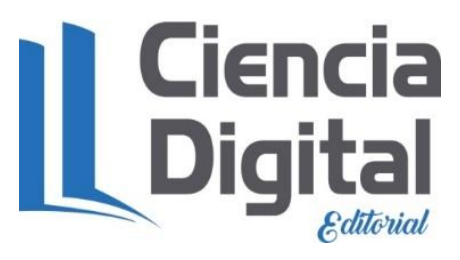

El artículo que se publica es de exclusiva responsabilidad de los autores y no necesariamente reflejan el pensamiento de la Revista Explorador Digital.

El artículo queda en propiedad de la revista y, por tanto, su publicación parcial y/o total en otro medio tiene que ser autorizado por el director de la Revista Explorador Digital.
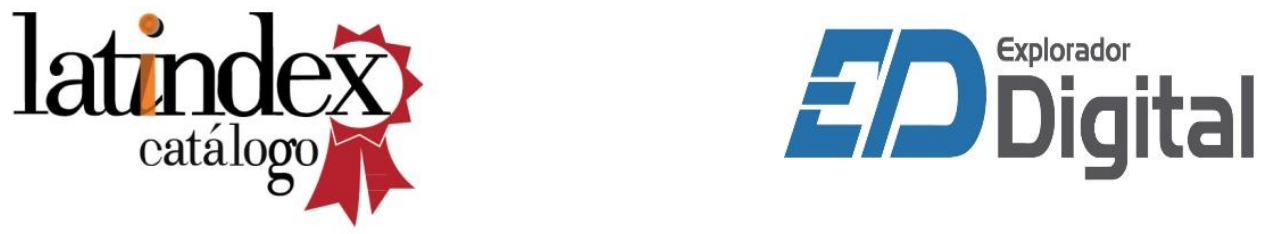